\title{
Drone Fleet Survivability Evaluation Based on Lanchester's Modified Deterministic Model
}

\author{
Herman Fesenko ${ }^{1}$, Vyacheslav Kharchenko ${ }^{1}$, Nikos Bardis ${ }^{2}$, Ah-Lian Kor ${ }^{3}$, Yevhen Brezhniev ${ }^{1}$ \\ ${ }^{1}$ National Aerospace University KhAI, 17 Chkalov str., 61070, Kharkiv, Ukraine, h.fesenko@csn.khai.edu, \\ v.kharchenko@csn.khai.edu \\ ${ }^{2}$ Hellenic Army Academy, Varis - Koropiou Avenue, Vari P.O. 16673, Athens, Greece \\ ${ }^{3}$ Leeds Beckett University, Headingley Campus, LS6 3QS, Leeds, United Kingdom A.Kor@leedsbeckett.ac.uk
}

Received: August 20, 2020. Revised: October 29, 2020, Accepted: November 5, 2020. Published: November 19, 2020.

\begin{abstract}
An algorithmic approach for the assessment of the survivability is proposed that is based on Lanchester's modified deterministic model. Methods are suggested for increasing the available time capability for nuclear power plant monitoring and coverage, using the required or a limited number of the operable drones,. Dependencies of the variance between the residual fleet damage and permissible drone fleet damage on monitoring time as well as dependencies of the monitoring time on the recovery group productivity are analysed.
\end{abstract}

Keywords-drone; survivability index; monitoring time; recovery group productivity; amazing factors; stability

\section{INTRODUCTION}

$\mathrm{I}^{\mathrm{N}}$ $\mathrm{N}$ recently years, drones, and other small unmanned aerial vehicles (UAVs) are actively used for the safe monitoring of radiation leakage and mapping of the affected areas in the case of Nuclear Power Plant (NPP) accidents. The advantages of the use of UAVs are apparent in comparison to manned aircraft [1]:

- Compact and agile equipment facilitates effective and timely reaction to events;

- Take-off and landing may be attempted at virtually any location and any time, without the need for licensed airfields or time - schedules;

- The existence and the state of the road network becomes irrelevant;

- The operator of the mission is able to remain in a safe location, at a safe distance from any contaminated areas;

- The cost of the platform equipment is lower compared to manned aircraft;

- Lower cost equipment may also be used for sampling and radiation measurements;

- The transportation of the equipment within the range required for the mission becomes easier;
- Possible contamination of the platform itself becomes irrelevant;

- Low overheads;

- The cost of fuel, as well as maintenance and service costs become insignificant.

The most detailed study on UAV survivability and reliability was published in 2003 [2]. According to [2], survivability of a UAV is its ability or component to avoid or withstand a hostile environment without suffering an abortive or catastrophic impairment of its ability to accomplish its designated mission, and reliability of a UAV is its ability and its parts to perform its mission without failure, degradation, nor demand on the support system. It is depicted as a percentage which represents the probability that a system or component will operate failure-free for a specified time, typically the throughout the mission duration.

\subsection{Problem Statement}

The UAVs, such as drones, have some disadvantages that can make the post-accident NPP monitoring mission impossible [3]:

- Human error in the remote controls can cause a drone to crash;

- Drones are sensitive to high winds and precipitation;

- Computer malfunction could occur resulting in the loss of control of a drone;

- Computer systems or software could break down resulting in loss of a drone and/or casualties on the ground;

- A drones or drone fleet can be hijacked by terrorists.

Also note that during its missions, a UAV is susceptible to various perturbations that cause disruptions to the mission schedule, operational failures, long term damage and an increase of the accident risk level. Such perturbations may include [4]:

- Strong winds during the time of flight, that cause significant deviations from the flight path and sudden changes in the orientation of the aircraft;

- External forces and moments acting upon the UAV that are not consistent with design assumptions; 
- Regression of the inertial characteristics after the release of the cargo;

- Damage to the fuselage, the wings and other body parts of the aircraft due to accidental collisions;

- Extreme force distribution states during transitions from hover mode to horizontal flight, and vice versa, leading to controllability breakdown;

- Mid-air collisions resulting in abrupt flight trajectory changes.

Electronics failures account for about $25 \%$ of all UAV failures [5]. There exists a variety of factors that may cause failure of the electronic equipment. Such factors include electrostatic discharges, power surges, vibrations that gradually degrade the functionality of soldering connections and joints and thermal fatigue of interconnections that is caused by material properties such as the coefficient of thermal expansion.

The existence of the above-mentioned inherent problems, mission perturbations, risks of electronic equipment failure, as well as a wide range of other uncontrollable conditions encountered at NPP accident sites, render necessary the ability to estimate and plan the required level of a drone fleet survivability.

\subsection{Aim and structure of the paper}

This research uses a modified version of Lanchester's deterministic model in order to derive a model for drone fleet survivability. The fleet is considered as a recoverable multi-state system and salient conditions of NPP accidents are incorporated.

Related work on drone fleet use for various tasks, including disaster and post accident monitoring, is considered in Sect. II. Drone fleet management issues and approaches to drone fleet survivability and reliability assessment are considered as well.

Methodology of evaluating the recoverable drone fleet survivability based on Lanchester's modified deterministic model is proposed in Sect. III. The main part of the methodology is an algorithm for evaluating the drone fleet survivability index.

Sect. IV presents and discusses results on the drone fleet survivability assessment by means of the algorithm proposed in Sect. III.

General conclusions are discussed in Sect. V. The conclusions cover ways for increasing NPP monitoring time using the recoverable drone fleet and future work on drone fleet survivability assessment.

\section{RELATED WORK}

\subsection{Drone Fleet General Applications}

Possible general drone fleet applications include emergency response [6]-[9], traffic control tasks [10], soil fertility and crop management [11], forest protection and wildfire monitoring [12], energy and electrical facility monitoring [13], pipeline inspection [14], and coast guard support [15].

\subsection{Drone Fleet and Disaster Monitoring}

The paper [6] proposes a drone-based solution to support search and rescue activities in disaster situations. The proposed architecture is composed of specialized drones to accomplish specific tasks and internal modules organization to accomplish their respective objectives. Among the advantages drones may bring to an accident monitoring, authors [7] highlight the gain in terms of time and human resources, as they can free rescue teams from timeconsuming data collection tasks and assist research operations with more insightful and precise guidance thanks to advanced sensing capabilities. Tina et al. [8] present a novel approach of using UAVs to establish a communication infrastructure in disaster situations. They describe in detail, three components of the system: end-toend communication, localization and navigation, and coordination. An approach based on the extent of interaction between the UAV and terrestrially deployed wireless sensors is presented in [9].

\subsection{Drone Fleet and Nuclear Power Plant Post Accident Monitoring}

In the opinion of the designers (for a new UAV Supercam S-350 which has been designed for meteorological and environmental monitoring in Russia), it is imperative for drone fleets [16] to be capable of monitoring radiation levels over specified areas. Elements of such missions include: outlining of nuclear and radiation accident emergency sites and measuring the levels of contamination; detection of radioactive sources and radioactive plume movement; examination of nuclear facilities for standards compliance; monitoring ground and air for dispersed radioactive pollutants; scientific research capabilities; initial assessment of radiation levels of an area, in the context of mobile radiation monitoring labs; capabilities for the rapid detection of x-rays and gamma emission sources etc. Aerial radiation monitoring has been the object of extensive research for a significant period of time and continues to attract the interest of researchers [17], with three-dimensional (3D) radiation mapping being within current research aims. The combination of high-resolution 3D topography combined with radiation surveying provides a powerful instrument for perceiving, measuring, monitoring and managing radiological contamination within a site both for radiological leakage events and routine site monitoring. An example of such an approach is presented in [18] whereby a scalable and adaptable sensor system is presented that is capable of performing a range of airborne operations including reconnaissance and radiological and nuclear surveillance. A remote sensing system is presented in [19] suitable for detecting radiation and acquiring aerial imaging. A simulated contour mapping of the nuclear radiation is used in [20] in order to evaluate two scenarios for the detection of nuclear radiation, incorporating multiple UAVs. An affordable UAV design is also given with an inherent cluster formation control and standard flight testing. A drone utilization method is given in [21], for examining both contaminated and remediated areas, with minimal exposure of the operator, for application in areas normally inaccessible by terrestrial survey methods. An affordable, compact, unmanned aerial platform was proposed by MacFarlane et al. [22] equipped with a embedded processor, an integrated gamma spectrometer, GPS and LIDAR. The device is demonstrated to be capable of rapidly and remotely detecting ground-based radiation patterns with a high spatial resolution $(<1 \mathrm{~m})$.

In [23], the unmanned drone cluster for radioactivity monitoring of a contaminated sites remotely (e.g. 
conducting tests in Chernobyl NPP exclusion zone). To enhance the features of post-accident monitoring systems, the principles of creating supplementary mobile ad-hoc wireless communication network for sensors data collection based on repeaters located on drones are presented in [24], [25].

\subsection{Drone Fleet Management Model}

The paper [26] proposes a fleet managing model for VTOL-UAV drones (Vertical TakeOff and Landing Unmanned Aerial Vehicle) that perform the delivery or pickup of supplies and materials in a production plant. Additionally, it also incorporates alternation in drone operations for an efficient use of energy in their batteries.

According to [24], [25], drone fleet can be categorized based roles and equipment employed: repeaters, observers (equipped with a TV camera), and additional sensors (they can be located in drones or be dropped down in certain places). Drones should be able to change their role through upgraded equipment at the location base. Drone-repeaters work together on a principle of "one leader". If the "leading drone-repeater" (Master) is damaged then other dronerepeater takes over the Master functions. An example of a principle applied for the choice of this substitute Master is a drone with the lowest working time (among all existing drones in the operational fleet) at the accident place.

In [27], we can find a list of companies that propose several drone fleet management systems. For example, Skycatch (San Francisco, United States) allows its users to deploy flying autonomous drones with real-time HD imagery and video from the sky; Garuda Robotics [Palo Alto, United States] proposes a platform that enables users to control mixed fleets without the expense of building and maintaining custom software and hardware; DroneDeploy (San Francisco, United States) is a smart drone management platform that helps users get stuff done with drones. Flirtey (Sydney, Australia) presents UAVs as a speedier, costeffective and environmentally friendly alternative to traditional delivery methods. PixiePath (Santa Barbara, United States) proposes a cloud-based drone fleet management platform. Its software handles drone movement in real time, and it is all browser compliant so it can be accessed from any connected device. It also helps pilots monitor their drones' battery levels, locations, and tasks.

\subsection{Drone Fleet Survivability and Reliability Assessment}

The problem of monitoring the NPP accident zone during the period after the event is examined in [28] with drone clusters are considered as systems with multi-level degradation. System Reliability Block Diagrams are used. The analysis includes system degradation levels, formulae for system reliability functions at these levels and conditions determining the levels.

A general structure and underlying principles for creating an Internet-of-Drone-based multi-version post-severe NPP accident monitoring system is described in [29]. The proposed design consists of an Internet-of-Things subsystem, a single-wired subsystem, and three drone-based wireless subsystems. Reliability block diagrams for the system and its subsystems are built based on considerations of different variants of sensor, communication, and decision-making systems. On the basis of reliability block diagrams, reliability models of the system and their subsystems are developed.
The work [30] deals with the reliability modeling and assessing of a mechatronic subsystem used for an UAV flight control with a triple modular redundancy. Markov chains and Reliability Block Diagrams are used for graphic representation of the proposed model.

A matrix of drone fleet reliability assessment attributes is presented in [31].

A structure of a multi-fleet of drones, consisting of main drone fleets and a reserve drone fleet is considered in [32]. In accordance with the structure, reliability models for centralized (irredundant), centralized (redundant), decentralized, and partially decentralized systems of control stations for the multi-fleet of drones are suggested and analyzed. The reliability models are described by means of reliability assessment attributes, presented in [31].

The papers [32-34] discuss the necessary steps for implementing the survivability concept for modern UAV and approach to reliability and survivability assessment.

\subsection{Summary}

A drone fleet used for NPP post accident monitoring can be categorized according to their roles and equipment: repeaters, observers and additional sensors.

There are many companies that propose several drone fleet management systems: Skycatch (San Francisco, United States); Garuda Robotics [Palo Alto, United States]; DroneDeploy (San Francisco, United States), Flirtey (Sydney, Australia), PixiePath (Santa Barbara, United States).

Fault Tree Analysis, Structure Function, Binary Decision Diagram, Markov chains and Reliability Block Diagrams are approaches used for drone reliability assessment.

\section{Methodology}

\subsection{Introduction}

The methodology of assessing drone fleet survivability is based on the application of a modified version of Lanchester's deterministic model. In this paper, a suggested algorithm is applied step by step for the evaluation of fleet survivability index considering recovery parameters.

\subsection{Simulation}

\subsubsection{Introduction to the simulation employed for this research}

It is assumed that the drones of a fleet used for NPP post accident monitoring perform the tasks (collecting information from control posts, hovering over specified terrain points) with a given level of quality (occupy the specified degradation level) during the given time $\tau_{g}$.

\subsubsection{Underpinning theory and principle}

If the quality of the tasks for drone fleet is determined by the number of operable drones in the fleet, it is necessary to ensure the presence of at least $m$ operable drones (in a fleet), with an initial number of drones represented by $n_{0}$ and required monitoring time $\tau_{g}$. The variable $i=1, \ldots n$, where $n$ is the number of degradation levels of the drone fleet for a given time $\tau_{g}$, which can be determined as follows:

$$
n\left(\tau_{g}\right)=n_{0}-m+1
$$




\subsubsection{Metrics for drone survivability index}

The main metrics for drone survivability index are:

- number of degradation levels of the drone fleet $n$ (1);

- variance between the residual fleet damage and permissible drone fleet damage $\Delta D$;

- Boolean drone fleet survivability index, $G$ which is a function of $\Delta D$.

\subsubsection{Input parameters}

Let us introduce the following parameters needed for evaluating the drone fleet survivability. They are tabulated in Table 1.

\begin{tabular}{|l|l|}
\hline \multicolumn{1}{|c|}{ Parameters } & \multicolumn{1}{c|}{ Description } \\
\hline$n_{0}$ & initial number of the drones \\
\hline$\tau$ & NPP monitoring time (h) \\
\hline$\tau_{g}$ & $\begin{array}{l}\text { mequired NPP monitoring time (h) } \\
\text { for the NPP monitoring }\end{array}$ \\
\hline$m$ & $\begin{array}{l}\text { number of drones in a drone fleet group } i \\
\text { which is expected to be exposed to } \\
\text { salient factors during the required } \\
\text { monitoring time } \tau_{g}\end{array}$ \\
\hline$n_{i}$ & $\begin{array}{l}\text { drone stability of a drone fleet group } i \\
\text { which is expected to be exposed to } \\
\text { salient factors during the required } \\
\text { monitoring time } \tau_{g}\end{array}$ \\
\hline$M$ & number of means to restore the drones \\
\hline$U_{i}$ & $\begin{array}{l}\text { productivity of a recovery group } i \\
\text { (drones per hour (dr/h)) }\end{array}$ \\
\hline$\lambda$ & drone fleet failure rate \\
\hline$\mu$ & drone fleet restoration rate \\
\hline
\end{tabular}

Table 1: Parameters for the Experiments

\subsubsection{Data source for the model}

Data for the model was prepared in accordance with recommendation presented in [35].

\subsubsection{Limitations and assumptions}

Assume the following limitations and assumptions.

1. The Lanchester's model with the logarithmic law of defeat [7] is used:

$$
\frac{d n}{d \tau}=-\lambda n+\mu \mathrm{n},
$$

where $\lambda$ is drone fleet failure rate, $\mu$ is drone fleet restoration rate. Taking into account the initial conditions $n(\tau=0)=n_{0}$ (the initial number of the drones), the solution of (2) is (3).

$$
n(\tau)=n_{0} e^{-(\lambda-\mu) \tau}
$$

2. The drone fleet is recoverable.

3. The drone fleet is exposed to amazing factors of the NPP accident.

\subsection{Algorithm for building the model}

The algorithm of evaluating the drone fleet survivability index includes the following steps:

1. Determination of the monitoring time during which it is possible to accept the assumption that both the failure and restoration rates of the drone fleet are constant;

2. Calculation of the average value of the drone fleet restoration rate

$$
\mu=\frac{1}{n_{0}} \sum_{i=1}^{M} U_{i}
$$

If the drones are restored by one recovery group $(\mu=1)$, expression in (4) can be converted to a simpler form:

$$
\mu=\frac{U}{n_{0}}
$$

3. Calculation of the approximate value of the drone fleet failure rate

$$
\lambda=\frac{1}{\tau_{g} n_{0}} \sum_{i=1}^{N} n_{i}\left(1-q_{i}\right)
$$

If the drones have the same stability $\left(q_{i}=q\right)$ and all the drones are exposed to salient factors, (6) can be presented as

$$
\lambda=\frac{1-q}{\tau}
$$

4. Calculation of the number of the drones that remain in an operable state during the monitoring time $\tau \leq$ $\tau_{g}$

$$
n(\tau)=n_{0} e^{-(\lambda-\mu) \tau}
$$

5. Calculation of the drone residual fleet damage

$$
D_{r}(\tau)=n_{0}-n(\tau)
$$

6. Calculation of the permissible drone fleet damage

$$
D_{p}(\tau)=n_{0} e^{-(\lambda-\mu) \tau}-m
$$

7. Calculation of the variance between the residual fleet damage and permissible drone fleet damage

$$
\Delta D=D_{r}(\tau)-D_{p}(\tau)
$$

8. Calculation of the Boolean drone fleet survivability index

$$
G=\left\{\begin{array}{l}
1, \text { if } \Delta D \leq 0 \\
0, \text { if } \Delta D>0
\end{array}\right.
$$

\subsection{Summary}

The algorithm of evaluating the drone fleet survivability index has been proposed. It is assumed that the drones of the 
fleet used for NPP post accident monitoring perform the following tasks: collecting information from control posts, hovering over specified terrain points.

\section{RESUlTS AND DisCUSSION}

\subsection{Introduction}

The main aim of this Section is to build and analyse the following dependencies: dependencies of the variance between the residual fleet damage and permissible drone fleet damage on the NPP monitoring time and dependencies of the NPP monitoring time on the recovery group productivity.

\subsection{Research results}

Experiment Set 1. Assume the recovery group productivity $U=0$. Based on the proposed algorithm of evaluating the drone fleet survivability index, change the NPP monitoring time to $\tau$. The rest of the parameters remain constant (drone stability $q=0.2$, required NPP monitoring time $\tau_{g}=12 \mathrm{hrs}$, initial number of drones in the fleet, $n_{0}=$ 10 , the minimum number of the drones needed for the NPP monitoring $m=4$ ). The NPP monitoring time $\tau$ are: 4: 6; 8; $10 ; 12 \mathrm{hrs}$. Repeat these actions for cases when the recovery group productivity $U=0.2 \mathrm{dr} / \mathrm{h}$ and $0.4 \mathrm{dr} / \mathrm{h}$ (note $\mathrm{dr} / \mathrm{h}$ denotes drones per hour).

Dependencies obtained according to Experiment Set 1 are shown in Fig 1.

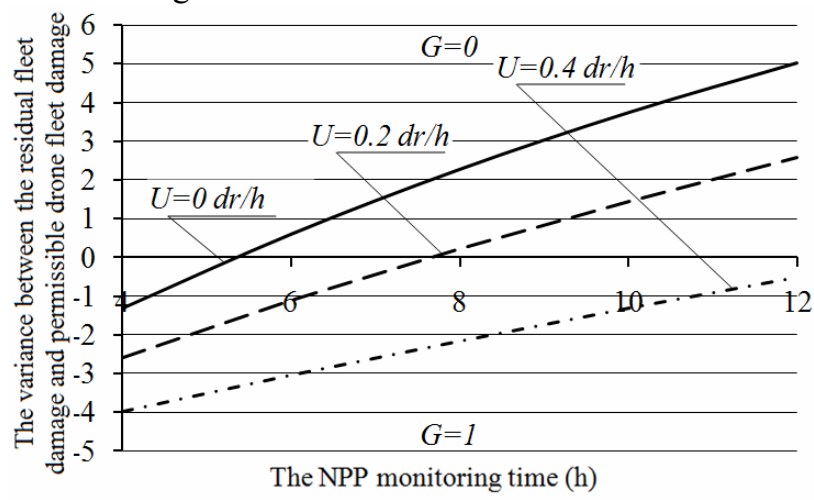

Figure 1. Dependencies of the variance between the residual fleet damage and permissible drone fleet damage on the NPP monitoring time

From Fig. 1, we can see that only for the recovery group productivity $U=0.4 \mathrm{dr} / \mathrm{h}$, the plot of the function $\Delta D=f(\tau)$ is entirely located in the negative plane, marked by " $G=1$ ". This means such recovery group productivity ensures at least four operable drones are required in the required monitoring time $\tau_{g}=12 \mathrm{~h}$. If any drones are not restored ( $U$ $=0 \mathrm{dr} / \mathrm{h}$ ), the monitoring time $\tau$ using at least four required operable drones will be reduced to $5.4 \mathrm{~h}$. If the recovery group productivity $U=0.24 \mathrm{dr} / \mathrm{h}$, the drone fleet with at least four required operable drones will be able to perform its own tasks in the monitoring time $\tau=7.4 \mathrm{~h}$ only. The last two values of the monitoring time $\tau$ can be obtained both graphically from Fig. 1 and using (13).

$$
\begin{aligned}
& \tau=-\frac{1}{\left(\frac{1-q}{\tau_{g}}-\frac{U}{n_{0}}\right)} \ln \left(\frac{m+n_{0}}{2 n_{0}}\right)= \\
& =-\frac{1}{(\lambda-\mu)} \ln \left(\frac{m+n_{0}}{2 n_{0}}\right)
\end{aligned}
$$

Experiment Set 2. Assume the minimum number of drones needed for the NPP monitoring $m=4$. Using (13), change the recovery group productivity $U$. The rest of the parameters remain constant (drone stability $q=0.2$, required NPP monitoring time $\tau_{g}=14 \mathrm{hrs}$, the initial number of the drones $n_{0}=10$ ). The recovery group productivity $U: 0 \mathrm{dr} / \mathrm{h}$; $0.15 \mathrm{dr} / \mathrm{h} ; 0.25 \mathrm{dr} / \mathrm{h} ; 0.3 \mathrm{dr} / \mathrm{h}$. Repeat these actions for cases when the minimum number of the drones needed for the NPP monitoring $m=5$ and 6 .

Dependencies obtained according to Experiment Set 2 are presented in Fig 2.

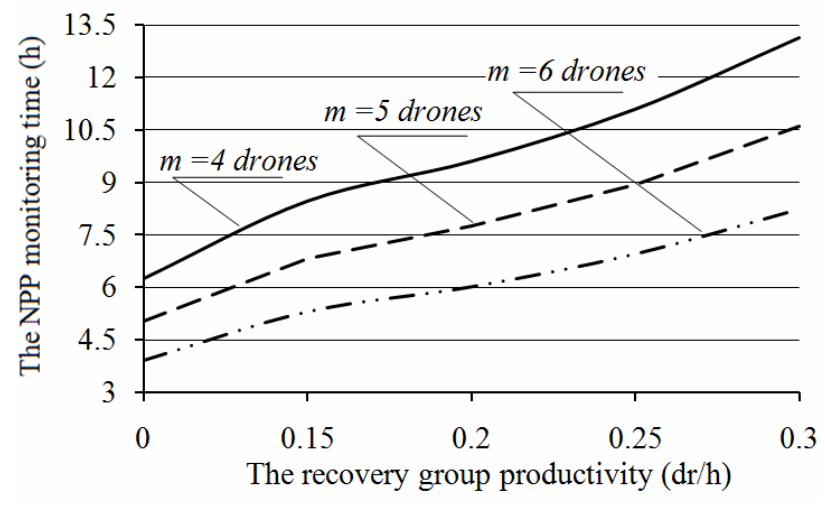

Figure 2. Dependencies of the NPP monitoring time on the recovery group productivity

We can make the following conclusions based on the analysis of the dependencies shown in Fig. 2.

1. There are two ways to increase the monitoring time $\tau$. The first one is to increase the recovery group productivity. The second one is to decrease the minimum number of the drones needed for the NPP monitoring. The last approach is be possible by: extending the drone flight range, applying more sophisticated equipment for the NPP monitoring, etc.

2. The maximum value of the monitoring time $\tau$ is achieved when the recovery group productivity $U=$ $0.3 \mathrm{dr} / \mathrm{h}$ and minimum number of drones required for performing the required tasks $m=4$ (Fig. 1), whereas the minimum value of the NPP monitoring time $\tau_{g}$ is achieved when no drone is restored (the recovery group productivity $U=0 \mathrm{dr} / \mathrm{h}$ ) and the minimum number of drones required for the NPP monitoring $m=6$.

\subsection{Summary}

Thus dependencies of the variance between the residual fleet damage and permissible drone fleet damage on monitoring time as well as dependencies of the monitoring time on the recovery group productivity are analysed. Based on the analysis of these dependencies and the ways for increasing the NPP monitoring time, employing the required number of the operable drones, is suggested. 


\section{CONCLUSIONS}

An approach and algorithm for drone fleet survivability assessment based on a stochastic continuous-time model have been proposed. According to the algorithm, the following parameters should be calculated:

- average value of the drone fleet restoration rate;

- approximate value of the drone fleet failure rate;

- number of drones that remain in operable state during monitoring time;

- drone residual fleet damage;

- $\quad$ permissible drone fleet damage;

- variance between the residual fleet damage; and permissible drone fleet damage;

- drone fleet survivability index.

Application of the algorithm makes it possible to determine ways for increasing the NPP monitoring time. Firstly, it involves increasing the recovery group productivity. Secondly, it entails decreasing the minimum number of the drones required for the NPP monitoring. This paper has demonstrated how the Dependencies of the variance between residual fleet damage and permissible drone fleet damage on the NPP monitoring time can be used to obtain the required recovery group productivity.

Future work will include tools on selecting the optimal number of redundant drones in drone fleet.

\section{References}

[1] Radiation surveillance and Unmanned Aerial Vehicles (2017, Jan.). [Online]. Available: https://www.stuk.fi/documents/88234/1051119/flyer_00 8 radiation_surveillance_and_uavs.pdf/ba8064c2-2428$485 \mathrm{c}-\mathrm{b} 4 \mathrm{e} 3-\overline{4} 0 \mathrm{f} 9042 \mathrm{c} 141 \overline{\mathrm{d}}$

[2] Office of the Secretary of Defense, "Unmanned Aerial Vehicle reliability study," 2003.

[3] Unmanned aerial vehicle uses, advantages and disadvantages. [Online]. Available: http://www.onlinesciences.com/robotics/unmanned-aerial-vehicle-usesadvantages-and-disadvantages

[4] A. Kulik, "Rational intellectualization of the aircraft control: Resources-saving safety improvement," in Green IT Engineering: Components, Networks and Systems Implementation, V. Kharchenko, Y. Kondratenko, J. Kacprzyk, Eds, vol. 151. Berlin. Heidelberg: Springer International Publishing, 2017, pp. 173-192.

[5] G. Caswell and E. Dodd. (2016, Aug.). Improving UAV reliability. [Online]. Available: http://cdn2.hubspot.net/hubfs/1871852/DfR_Solutions_ Website/Resources-Archived/White-

Papers/Reliability/Improving-Unmanned-AerialVehicle-UAV-Reliability.pdf?t=1482271815148

[6] D. Camara, "Cavalry to the rescue: Drones fleet to help rescuers operations over disasters scenarios," in Proc. 2014 IEEE Int. Conf. Antenna Measurements and Applicat., Antibes Juan-les-Pins, France, CAMA 2014, pp. 16-19.

[7] T. Tanzi, M. Chandra, J. Isnard, D. Camara, O. Sebastien, and F. Harivelo, "Towards drone-borne disaster management: Future application scenarios," in 2016 ISPRS Ann. Photogrammetry, Remote Sensing and Spatial Inform. Sci., Prague, Czech Republic, pp. 181189.
[8] G. Tuna, T. Veli, and K. Gulez, "Design strategies of unmanned aerial vehicle-aided communication for disaster recovery," in Proc. 9th Int. Conf. High Capacity Optical Networks and Enabling Technologies, Istanbul, Turkey, HONET 2012, pp. 115-119.

[9] M. Erdelj, E. Natalizio, K. Chowdhury, and I. Akyildiz, "Help from the sky: Leveraging UAVs for disaster management," IEEE Pervasive Computing, vol. 16, pp. 24-32, Jan. 2017.

[10] K. Kanistras, G. Martins, M. Rutherford, and K. Valavanis "Survey of unmanned aerial vehicles (UAVs) for traffic monitoring," in Handbook of Unmanned Aerial Vehicles, K. Valavanis and G. Vachtsevanos, Eds. New York: Springer Science+Business Media Dordrecht, 2015, pp. 2643-2666.

[11] K. R. Krishna. Push Button Agriculture: Robotics, Drones, Satellite-Guided Soil and Crop Management. Waretown, NJ: Apple Academic Press, Incorporated, 2016.

[12] L. Merino, F. Caballero, J. R. Martinez-de-Dios, I. Maza, and A. Ollero, "An unmanned aircraft system for automatic forest fire monitoring and measurement," Journal of Intelligent and Robotic Systems, vol. 65, no. 1, pp. 533-548, Jan. 2012.

[13] T. Roberts, B. Karaus, and D. Singh. (2016, Aug.). Use of drones for monitoring and inspection of energy infrastructure. [Online]. Available: http://www.vnf.com/use-of-drones-for-monitoring-andinspection-of.

[14] Pipeline inspections using drones. (2016, Nov.). [Online]. Available: http://advancedaerialservices.com/drone-videoinspection-and-survey-services/pipeline-inspection/

[15] Coast Guard wants marine drones for persistent surveillance. (2014, Apr.). [Online]. Available: https://defensesystems.com/articles/2014/04/08/coastguard-persistent-surveillance.aspx

[16] A new unmanned aerial vehicle for background radiation monitoring is developed for Russian meteorology and ecological monitoring corporation Rosgidromet. [Online]. Available: http://unmanned.ru/en/news/2014-06-02.htm

[17] D. Connora, P. G. Martin, and T. B. Scott, "Airborne radiation mapping: overview and application of current and future aerial systems," International Journal of Remote Sensing, vol. 37, no. 24, pp. 5953-5987, Nov. 2016.

[18] R. Pöllänen, H. Toivonena, K. Peräjärvia, T. Karhunen, T. Ilander, J. Lehtinen, K. Rintala, T. Katajainen, J. Niemelä, and M. Juusela, "Radiation surveillance using an unmanned aerial vehicle," Applied Radiation and Isotopes, vol. 67, no. 2, pp. 340-344, Feb. 2009.

[19] J. Towler, B. Krawiec, and K. Kochersberger, "Radiation mapping in post-disaster environments using an autonomous helicopter," Remote Sensing, vol. 4, no. 7, pp. 1995-2015, July 2012.

[20] J. Han, Y. Xu, L. Di, and Y. Chen, "Low-cost multiUAV technologies for contour mapping of nuclear radiation field," International ournal of Intelligent \& Robotic Systems, vol. 70, no. 1-4, pp. 401-410, Apr. 2013.

[21] P. G. Martin, O. D. Payton, J. S. Fardoulis, D. A. Richards, Y. Yamashiki, and T. B. Scott, "Low altitude unmanned aerial vehicle for characterising remediation 
effectiveness following the FDNPP accident," Journal of Environmental Radioactivity, vol. 151, pp. 58-63, Jan. 2016.

[22] J. W. MacFarlane, O. D. Payton, A.C. Keatley, G. P. T. Scott, H. Pullin, R. A. Crane, M. Smilion, I. Popescu, V. Curlea, and T. B. Scott, "Lightweight aerial vehicles for monitoring, assessment and mapping of radiation anomalies," Journal of Environmental Radioactivity, vol. 136, pp. 127-130, Oct. 2014.

[23] S. Babak, "Radiation Monitoring of Environment Using Unmanned Aerial Complex", The Avdanced Science Journal, 2014, issue 12, pp. 41-44.

[24] R. Hiromoto, A. Sachenko, V. Kochan, V. Koval, V. Turchenko, O. Roshchupkin, and K. Kovalok, "Mobile Ad Hoc wireless network for pre- and post-emergency situations in nuclear power plant," in Proc. IEEE Int. Symp. on Wireless Systems within the Conf. on Intelligent Data Acquisition and Advanced Computing Systems, Offenburg, Germany, IDAACS-SWS 2014, pp. $92-96$

[25] A. Sachenko, V. Kochan, V. Kharchenko, H. Roth, V. Yatskiv, M. Chernyshov, P. Bykovyy, O. Roshchupkin, V. Koval, and H. Fesenko, "Mobile post-emergency monitoring system for nuclear power plants," in Proc. 12th Int. Conf. ICT in Education, Research and Industrial Applications, Kyiv, Ukraine, ICTERI 2016, pp. 384-398.

[26] V. Olivares and F. Cordova, "Design of drone fleet management model in a production system of customized products," in Proc. 6th Int. Conf. Computers Communications and Control, Oradea, Romania, ICCCC 2016, pp. 165-172.

[27] Drones Sector Overview. March 2015. (2016, Aug.). [Online].

https://web.stanford.edu/group/cmc/cgi-

bin/wordpress/wp-

content/uploads/2015/11/DronesStartupLandscape_Apr -2015.pdf

[28] V. Kharchenko, A. Sachenko, V. Kochan, and H. Fesenko, "Reliability and survivability models of integrated drone-based systems for post emergency monitoring of NPPs," in Proc. IEEE Int. Conf. on
Information and Digital Technologies 2016, Rzeszow, Poland, IDT 2016, pp. 127-132.

[29] H. Fesenko, V. Kharchenko, A. Sachenko, R. Hiromoto, and V. Kochan, "An Internet of Drone-based Multi-version Post-severe Accident Monitoring System: Structures and Reliability," in Dependable IoT for Human and Industry Modeling, Architecting, Implementation, V. Kharchenko, A. Kor, A. Rucinski, Eds. Denmark, The Netherlands: River Publishers, 2018, pp. 197-217.

[30] C. Martin, V. Gonzalez-Prida, and F. Pérès, "Reliability assessment of a multi-redundant repairable mechatronic system," in Numerical Methods for Reliability and Safety Assessment: Multiscale and Multiphysics Systems, S. Kadry and A. Hami, Eds. Cham, Switzerland: Springer International Publishing, 2015, pp. 407-423.

[31] H. Fesenko, V. Kharchenko, and E. Zaitseva, "Evaluating Reliability of a Multi-fleet with a Reserve Drone Fleet: an Approach and Basic Model" in Proc. IEEE Int. Conf. Information and Digital Technologies, Zilina, Slovakia, IDT 2019, pp. 128-132.

[32] H. Fesenko and V. Kharchenko, "Reliability models for a multi-fleet of drones with two-level hot standby redundancy considering a control system structure," in Proc. 10th IEEE Int. Conf. Intell. Data Acquisition and Advanced Computing Syst.: Technology and Applicat., Metz, France, IDAACS 2019, pp.1-6.

[33] H. Fesenko, V. Kharchenko and N. Bardis "An approach to the drone fleet survivability assessment based on a combinatorial model," in Proc. Int. Conf. Mathematical Methods and Computational Techniques in Science and Engineering, Cambridge, UK, 2018, pp. $1-7$

[34] A. Muraru, "An overview on the concept of UAV survivability," in Proc. 13th Int. Conf. Scientific Papers "Scientific Research and Education in the Air Force", Brasov, Romania, AFASES 2011, pp. 1231-1236.

[35] Y. Stekolnykov, System survivability. St. Petersburg, Russia: Polytekhnyka press, 2002, 155 p. (in Russian).

\section{Creative Commons Attribution License 4.0 (Attribution 4.0 International, CC BY 4.0)}

This article is published under the terms of the Creative Commons Attribution License 4.0 https://creativecommons.org/licenses/by/4.0/deed.en_US 